\title{
Molten salt fast reactor SAMOFAR: Safety issues of the chemical plant
}

\author{
Anthony Marchix ${ }^{1 *}$ and Manon Dieuaide ${ }^{1}$ \\ ${ }^{1}$ IRFU, CEA, Université Paris-Saclay, F-91191 Gif-sur-Yvette, France
}

\begin{abstract}
The concept of Molten Salt Fast Reactor (MSFR) has been selecting by the Generation IV International forum as it presents interesting features in terms of safety, sustainability and economics. SAMOFAR project aims to provide the safety assessment of the MSFR concept based on the Thorium fuel cycle. This document provides the radiological assessments for the chemical plant, including shielding requirements and decay heat evaluations.
\end{abstract}

\section{Introduction}

Molten Salt Fast Reactor (MSFR) is one of the six fission reactor concepts that has been selected by the Generation IV International Forum [1] as it satisfies the goals of Generation IV reactors in terms of safety, sustainability, nonproliferation, waste management and economics. Within this framework, the project SAMOFAR (Safety Assessment of the Molten Salt Fast Reactor) aims to update the conceptual design, to deliver the experimental proof of concept of the safety features of the MSFR, to provide a safety assessment for both the reactor and the chemical plant and to deliver a roadmap for further exploitation of the project results [2]. The MSFR concept is based on the design developed by the CNRS Grenoble [3], which is characterized by a fast neutron spectrum, a fluoride liquid fuel and using the Thorium fuel cycle. This concept is based on the feedback of previous experiments and studies [4], and has the advantages to be operated as a breeder or burner mode, to purify the salt to reasonable rate $(40 \mathrm{~L} /$ day) compared to a moderated molten salt reactor $\left(4 \mathrm{~m}^{3} /\right.$ day), etc. In the following sections, the safety issues associated to the chemical plant are presented. This concerns the assessment of the radiological constraints associated to nuclides inventories involved in each chemical processes.

\section{Methodology}

To perform the safety assessment related to the chemical plant, a new tool has been developed that permits to compute the different inventories involved in any locations of the reprocessing unit. This tool is based on the Bateman equations that describes the time evolution of an inventory knowing the nuclear decay properties of its intrinsic constituents. Most of the fundamental properties that are needed to perform the safety assessments are obtained from experimental data, especially the nuclear decay properties and the chemical transfer coefficients.

*e-mail: anthony.marchix@cea.fr

\subsection{Chemical plant}

To ensure the neutronics properties (limitation of neutron absorbers) and to control the physicochemical properties of the salt (corrosion, deposit...), fission products must be extracted [5]. For this purpose, the chemical plant is composed of four main processes able to perform the chemical separation of the fission products from the fuel salt [68], i.e. the fluorination, the two reductive extractions and the actinide traps (green boxes in Figure 1). Every day, a batch of $40 L$ of fuel salt is extracted from the core for cleaning, called in the following core inventory. This inventory is first temporally stored to cool down before to pass through the different chemical processes. This step allows to limit the radiological constraints in the whole reprocessing unit as well as to limit as low as possible the nuclear transmutations that can occur during each chemical separation. Indeed, each chemical processes aim to extract a part of the fission products from the batch and to accumulate them in a specific storage area (orange boxes in Figure 1). At the end of the chemical loop, the fuel salt, including transthoric elements, have to be introduced back into the core.

The chemical plant contains three different storage areas, which are called gas, fission products and Lanthanides. In these areas, the associated inventories increase after each cycle as new elements are daily extracted and stored into. Concerning the inventories associated to the chemical processes (green boxes in Figure 1), every day the same amount of elements have to be considered for the safety assessment, which is only related to the core inventory and the transfer coefficients associated to the chemical processes of interest. Those transfer coefficients can be found in [9].

\subsection{Nuclear decay chains modelling}

To compute the time evolution of a nuclides inventory, the algorithm is based on the Bateman equations which provide the abundance of a specific nucleus within a decay 


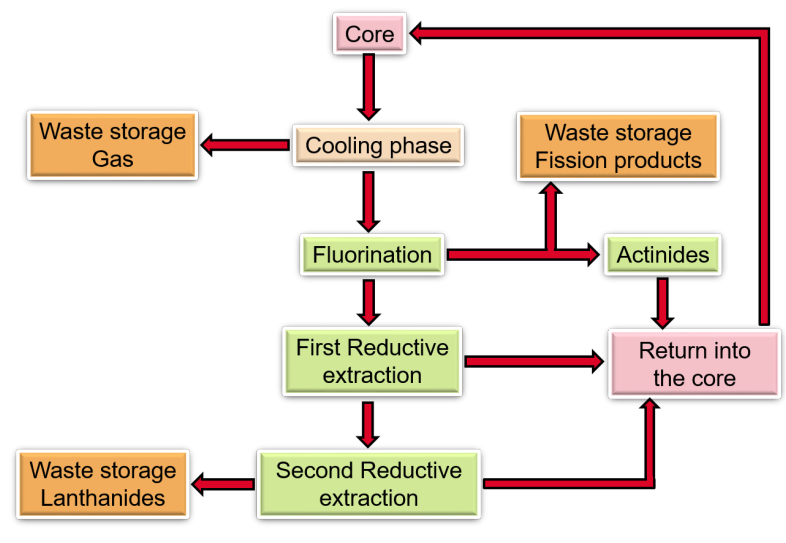

Figure 1. Scheme of the chemical plant

chain at a given time. The general form of the Bateman equations applied to an isotope $i$ within a radioactive chain takes the following form:

$$
\frac{d N_{i}(t)}{d t}=\sum_{j} \lambda_{j} N_{j}(t)-\lambda_{i} N_{i}(t)
$$

where $N_{i}(t)$ corresponds to the number of isotopes of type $i$ at a given time $t$, having a decay constant $\lambda_{i}$. This master equation stipulates that the time evolution of the number of isotopes $i$ is the balance between its own decay $\left(\lambda_{i} N_{i}(t)\right)$ and the summation of the decay of its mother isotopes $\left(\sum_{j} \lambda_{j} N_{j}(t)\right)$. Note that the nuclear transmutation induce by the neutron capture is not take into account as the neutron rate within the chemical plant, resulting from the spontaneous fissions and alpha-induced reactions, is extremely small $\left(\sim 10^{9}\right.$ neutrons. $\left.\mathrm{s}^{-1}\right)$ compared to the number of nuclear decays $\left(\sim 10^{18}\right.$ decays. $\left.\mathrm{s}^{-1}\right)$.

To solve this system of coupled differential equations, the algorithm determines all the different single decay chains that are initiated by each unstable isotopes of the core inventory. For a given single decay chain, the abundance associated to an isotope $i$ at time $t$ is given by the following analytical solution:

$$
N_{i}(t)=N_{0}(t=0) \prod_{k=0}^{i-1} \lambda_{k} \sum_{j=0}^{i} \frac{e^{-\lambda_{j} t}}{\prod_{l=0(l \neq j)}^{i}\left(\lambda_{l}-\lambda_{j}\right)}
$$

where $N_{0}(t=0)$ is the number of isotopes of the single decay chain initiator in agreement with its population within the core inventory. All its daughter isotopes are set to zero initially. In consequence, the whole inventory at a given time $t$ is simply the summation of the all $N_{i}(t)$ computed for all decay chains. To determine the single decay chains, as well as to compute the time evolution of nuclide inventories, radioactive decay information are extracted from the ENSDF (Evaluated Nuclear Structure Data File) database [10]. The following decay modes are taken into account: alpha, beta, electron capture, spontaneous fission, isomeric transition.

For instance, the core inventory is computed by assuming 200 years of operation time [11]. This assumption allows to perform conservative assessments as it provides a core inventory close to steady state conditions. A total of 1099 isotopes composes this inventory. The algorithm has defined 1551 single decay chains for which the abundances of daughter isotopes at a time $t$ are computed in agreement with the equation 2 .

\section{Discussion}

The evaluation of the safety issues aims to determine the radiological constraints associated to the chemical plant. A specific focus is done on the decay heat and the shielding requirements for each chemical processes. Nevertheless, the design of the chemical plant is not as advanced as the reactor, and only preliminary assessments can be done as the details associated to the chemical processes are not known (dimensions of inlet and outlet, size, etc) for conducting detailed studies. For instance, the cooling duration is not yet well defined but this determines the number of batches to temporally cool down within the chemical plant, and so the total amount of radioactivity mobilised into the chemical plant.

\subsection{Cooling phase}

The cooling phase aims to temporally store the $40 \mathrm{~L}$ batch for a specific duration, which has to be determined, in order to decrease the safety issues in the whole chemical loop and to improve the efficiency of the chemical processes. As the core inventory contains unstable isotopes, these latter will decay during their stay within the chemical plant. But, the chemical processes are efficient to extract isotopes with specific transfer coefficients. Nevertheless, those transfer coefficients are not necessary the same for an unstable isotope and its daughters. This is then important to limit as low as possible the radiological decay, especially for short half-life isotopes, to ensure that the chemical processes extract the right isotopes at the right location.

In Figure 2 is shown the impact of the cooling duration

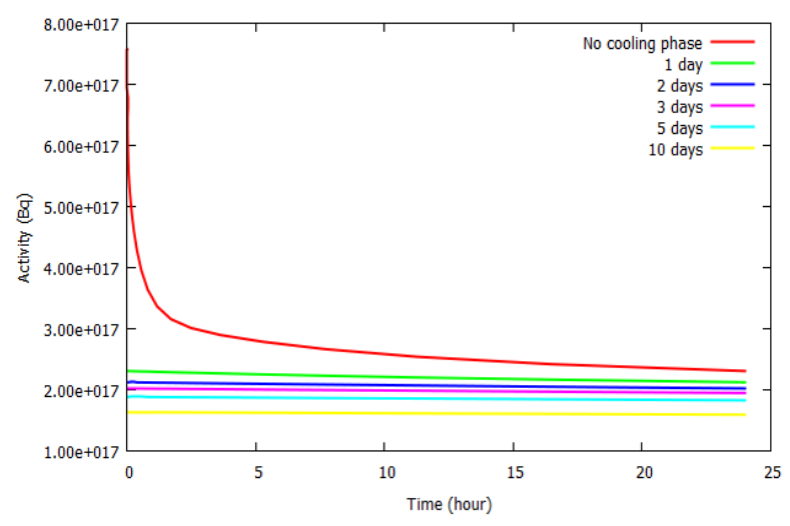

Figure 2. The total activity associated to the fluorination step as a function of time for different cooling durations

on the total activity of the $40 \mathrm{~L}$ batch at the first chemical process location, i.e. the fluorination step. When no cooling phase is considered, the total activity decrease 
strongly by a factor of 3 during the first day, mostly during the first 5 hours. This is due to the short half-life isotopes that decay during the time of the process (two hours for the fluorination step). The same observation can be done for all other chemical processes. Only for a cooling duration larger than one day, the total activity of the fuel salt is quite flat. After one day of cooling duration, the main contributors to the total activity are: ${ }^{233} \mathrm{~Pa}(62.1 \%),{ }^{141} \mathrm{Ce}(3.3 \%),{ }^{140} \mathrm{La}(3.3 \%),{ }^{95} \mathrm{Zr}$ (3.2\%). As shown, the total activity is completely dominated by the radioactive decay of ${ }^{233} \mathrm{~Pa}$, while the following contributors do not exceed more than $3.5 \%$. However, $40 \mathrm{~L}$ of fuel salt represents a total amount of isotopes of about 5167 moles while the number of unstable isotopes which decay during the first day is only about 0.04 moles . In Figure 3 is provided the time evolution of the number

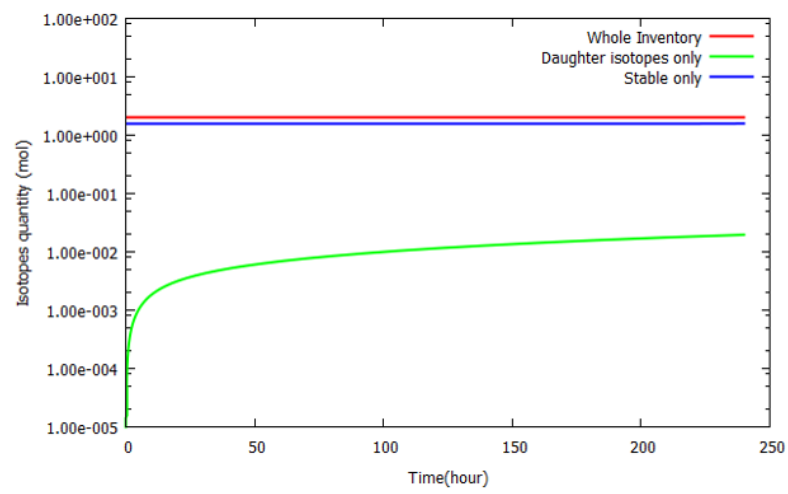

Figure 3. Number of isotopes stored in the fission products storage area as a function of the cooling duration

of isotopes daily stored into the fission products storage area. This total amount of isotopes is flat as a function of the cooling duration. Most of stored isotopes concerns stable isotopes, already present within the core inventory, that are distributed without any ambiguity by the different chemical processes and are extracted to the proper storage area. The same result is obtain for the Lanthanides storage area. In consequence, the cooling duration has no impact on the stored inventories.

The impact of the cooling duration on the decay heat is provided in Figure 4, where the decay heat associated to each chemical processes have been evaluated by considering one or two days of cooling time. Once the $40 L$ batch is extracted from the core, the decay heat is evaluated to about $180 \mathrm{~kW}$, mainly due to the beta decay $(55 \%)$. After one day of cooling duration, the decay heat is reduced to $23 \mathrm{~kW}$, and only $20 \mathrm{~kW}$ for two days. Concerning the storage areas, the decay heat is computed to about $2.2 \mathrm{~kW}$ and $6.3 \mathrm{~kW}$ for the fission products and the Lanthanides respectively for one day of cooling duration. Once cleaning, the fuel salt that is introduced back into the core still represents a decay heat of $14.8 \mathrm{~kW}$. This means that the decay heat dissipated within the salt and transferred to the surrounding components during the cooling phase is of $157 \mathrm{~kW}$. Within the storage areas, a daily contribution is added every day and the decay heat

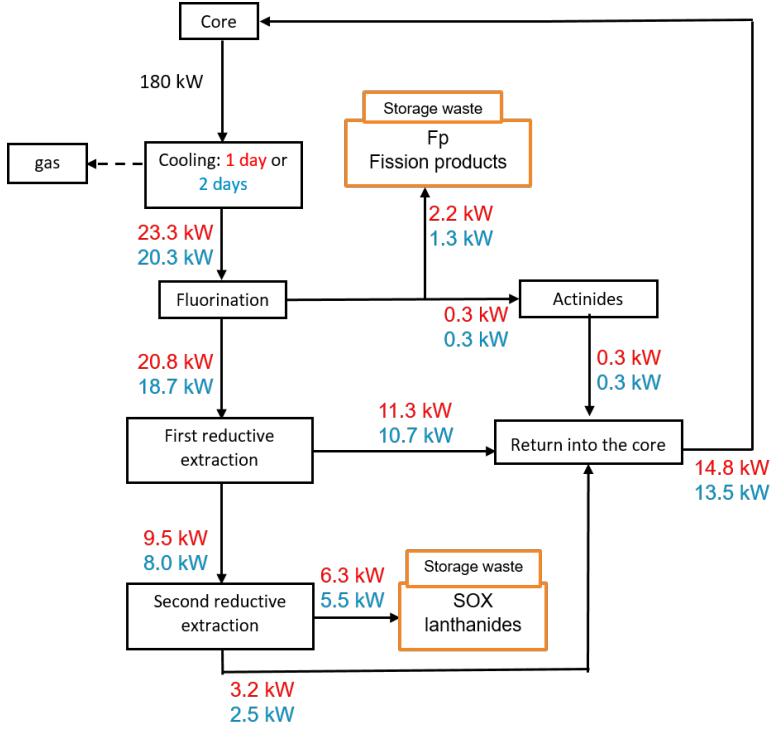

Figure 4. Decay heat evaluation within the chemical plant

is evaluated to $100 \mathrm{~kW}$ and $400 \mathrm{~kW}$ for the fission products and the Lanthanides storage areas respectively, taking into account an operation time of 500 days and one day of cooling duration. In consequence, the cooling phase has a strong impact on the decay heat within the chemical plant by focusing the constraint on the cooling phase and releasing them for all other chemical processes. The decay heat is significantly reduced (factor 8 ) by considering one day of cooling duration.

\subsection{Shielding assessments}

To assess the shielding requirements, the gamma and the neutron source terms have been evaluated. The former one is directly deduced from the nuclear database ENSDF by considering the rate and the gamma energy associated to the gamma transition following the beta and the alpha decays. About the neutron source term, the alpha source term has first to be evaluated by using also the nuclear database ENSDF so as to perform the $(\alpha, n)$ reactions. In the following, only gamma shielding requirements is discussed as it concerns the most constraining source term for shielding requirements. As no detailed information are available on the dimensions of the chemical processes, a simple geometry has been considered: the source is modelised by a sphere corresponding to a volume of $40 \mathrm{~L}$ of fuel salt, surrounding by a sphere for the gamma shielding made of concrete or lead. The shielding thickness is adjusted to the radiological requirements, expressed in terms of equivalent dose rate.

In Figure 5 is shown the impact of the cooling duration on the gamma spectrum defined at the most constraining location, i.e. the cooling phase. After one day of cooling duration, the high-energy tail of the gamma specrum is strongly reduced due to the decay of short half-life isotopes. Less energetic gamma help to reduce the shielding thickness. In the following, the gamma sheilding for 


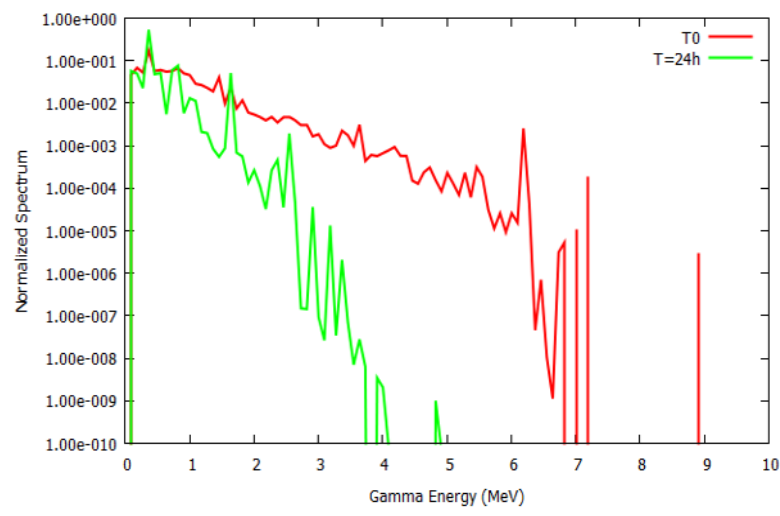

Figure 5. Gamma spectrum for two cooling durations ( $\mathrm{O}$ and 1 day)

the different chemical processes is done by assuming a cooling duration of one day.

In Figure 6 is shown the concrete shielding associated

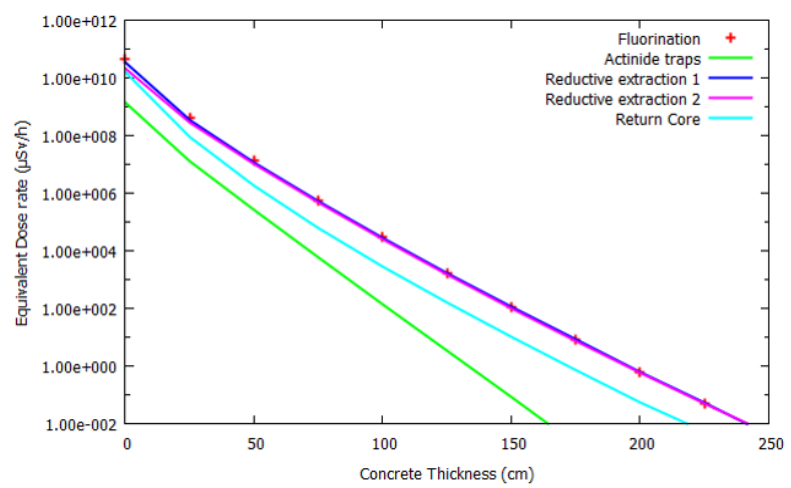

Figure 6. Concrete shielding associated to the gamma radiations

to the gamma source term of each chemical processes. The most contraining inventories, in terms of equivalent dose rate, concern those associated to the fluorination and the two reductive extraction processes. In order to reach an equivalent dose rate of $0.5 \mu S v / h$, a concrete shielding of about $200 \mathrm{~cm}$ is needed. This corresponds to a lead thickness of about $40 \mathrm{~cm}$. Of course, the most constraining radiation source for the shielding requirement is the gamma source associated to the cooling phase that requires $325 \mathrm{~cm}$ of ordinary concrete or $55 \mathrm{~cm}$ of lead to reach an equivalent dose rate of $0.5 \mu \mathrm{S} v / h$. For the storage areas, the lead shielding have been evaluated in view of the handling of the stored inventories. This evaluation depends on the dose level requirements related to the transportation package and associated regulation. To limit the dose rate at contact to less than $25 \mu S v / h$, one needs a lead thickness of about $30 \mathrm{~cm}$ and $37 \mathrm{~cm}$ surrounding the container of the Fission products and the Lanthanides respectively for an operation time of 2000 days.

\section{Conclusion}

A tool has been developed, based on the Bateman equations, in order to treat the evolution of inventories associated to each process involved in the chemical plant. Safety evaluations are based on experimental data, especially the nuclear decay properties and the chemical transfer coefficients. A preliminary analysis have been performed on the cooling phase to determine the cooling duration that allows to reduce the constraints on the safety observables. It has been highlighted that one day permits to limit the decay heat within the chemical loop, as well as the reduce the high-energy tail of the gamma spectrum. A view of the gamma shielding requirement has been done for all chemical processes.

Authors want to thank S. Delpech, G. Duran-Klie, D. Rodriguez from CNRS Orsay and E. Merle, D. Heuer from CNRS Grenoble for fruitful discussions. SAMOFAR has received funding from EURATOM research and training program 2014-2018 under grant agreement No 661891.

\section{References}

[1] GIF, (Generation IV International Forum), Annual report (2008)

[2] J.L. Kloosterman et al., Molten Salt Reactors and Thorium Energy, 565-570 (2017)

[3] E. Merle-Lucotte et al., Proceedings of the ThEC13 Conference, CERN, Globe and Science Innivation, Geneva, Switzerland, 223-231 (2013)

[4] J. Serp et al., Prog. in Nuc. Ener. 77, 308-319 (2014)

[5] M. Allibert et al., Handbook of Generation IV Nuclear Reactors, 157-188 (2016)

[6] S. Delpech et al., J. of Fluorine Chemistry 130, 11-17 (2009)

[7] S. Delpech et al., Proceedings of the ThEC13 Conference, CERN, Globe and Science Innivation, Geneva, Switzerland, 177-183 (2013)

[8] D. Rodrigues et al., NUKLEONIKA 60, 907-914 (2015)

[9] S. Delpech, Pure Appl. Chem. 85, 71-87 (2013)

[10] J.K. Tuli, National Nuclear Data Center, Brookhaven National Laboratory, BNL-NCS-51655-01/02-Rev, 2001, http://www.nndc.bnl.gov/ensdf

[11] D. Heuer et al., Ann. of Nuc. Ener 64, 421-429 (2014) 